\title{
Cerebral venous sinus thrombosis: Clinical profile and surgical outcome
}

\author{
Bhagwati Salgotra $^{1, *}$, Dhruv Patel ${ }^{2}$ \\ ${ }^{\mathbf{1}}$ Associate Professor, ${ }^{2}$ Senior Resident, Dept. of Neurosurgery, Sumandeep Vidhyapeeth Dhiraj Hospital, Vadodara, Gujarat, \\ India
}

*Corresponding Author:

Email: drsalgotra@gmail.com

\begin{abstract}
Thrombosis of the cerebral venous sinuses (CVST) is common cause of acute neurological deficit and altered sensorium. Most of the patients are young and can suffer severe morbidity and mortality if not treated aggressively. Early diagnosis and management is necessary for good outcome in case of CVST.

Material and Methods: Retrospective observational study over a period of 4 year 3 months from oct' 2013 to feb'2018 in our institute. CVST was affirmed by MRI brain venogram of 84 patients were included in this study. Decompressive craniectomy done in case of mass effect or herniation. All patients were treated according to standard guideline and protocol.

Result: This study include $51(60.7 \%)$ females and $33(39.2 \%)$ males with the age of $39 \pm 10$ years and $29 \pm 7$ years, respectively. Out of which 25 patients $(29.7 \%)$ had hemiparesis/hemiplegia, vomiting and headache was manifested in 79 (94\%) patients, seizures in 13(15\%), and fever in 30(35.7\%). 38(45.2\%) out of 84 patients were presented with sagittal sinus thrombosis, $10(11.9 \%$ ) of 84 with multiple sinus thrombosis and 35(41.6) of 84 patients with transverse/sigmoid sinus thrombosis. On funduscopic examination papilledema in $25(29.7 \%)$ patients. Patients had diagnosis of CVST during peripartum period altogether $8(15.6 \%)$ out of 51 female. 1 patient had bilateral cavernous sinus thrombosis. Signs of infection seen in patients in the study as Chronic supportive otitis media (CSOM), fever, 11(13.09\%) patients had paranasal sinus (PNS) infections, $10(11.9 \%)$ patients had evidence and history of lack of hydration. $8(9.5 \%)$ patients of the total deceased during the course of treatment and $79(94 \%)$ out of 84 patients were discharged with partial or/and total recovery. Three 25(29.7\%) required decompressive craniectomy. Multiple sinus thrombosis had poor prognosis.

Conclusion: Most commonly involved sinus is superior sagittal sinus while transverse or sigmoid sinus and bilateral cavernous sinus thrombosis follows it being uncommon fondness in CVST.' Third part of the female population was influenced because of oral contraceptive pills and the peripartum period has high risk for CVST'. The lack of hydration and/or infection was the most commonly related accelerating event for the advancement of CVST and hyper homocysteinemia which is seen in more than one fourth of the population. Mortality and morbidity was more in patients of multiple sinus involvement. The treatment must be aggressive as mortality and morbidity is relatively minimal compared with the arterial stroke.
\end{abstract}

Keywords: Cerebral venous sinuses thrombosis, Decompressive craniectomy, Thrombosis of sagittal sinus.

\section{Introduction}

In younger age group, Cerebral venous sinus thrombosis (CVST) is one of the common causes of stroke. Young to moderately aged individuals are usually affected by CVST with potentially grave consequences and in India nearly $30 \%$ of all stroke cases are accounted in youngsters and $10-20 \%$ of these cases represent cerebral venous thrombosis (CVT).

"Of all the types of stroke CSVT represents almost $0.5 \%{ }^{2}-3 \%,{ }^{2}$ influencing predominantly younger age group, ${ }^{3}$ with an estimated incidence for adults of 3-4 per million, and for children 7 per million. ${ }^{4}$ In the preanti-microbial time, the main cause of CSVT were the septic procedures, currently the aseptic form of CSVT is the most widely recognized cause". ${ }^{5}$ CSVT is characterized by variable etiologies, difficult diagnosis, and prognosis. " 11.6 cases out of 100000 deliveries have been established with the peripartum-associated CSVT. ${ }^{7}$ There is sex predominance of women with $75 \%$ of all CSVT patients, with a ratio of three is to one compared to men." 8

A varied number of state and disorders are responsible for CVST including prothrombotic states and disorders of clotting system such as inherited cause is Protein $\mathrm{C}$ resistance optional to Factor $\mathrm{V}$ Leiden polymorphism, lack of antithrombin III and Protein C and $\mathrm{S}$ resistance. Other vasculitis such as polyarteritis nodosa (PAN) and Systemic lupus erythematosus (SLE) are likewise pertinent in youthful grown-ups. 0.5\%-3\% of CVST is responsible for all strokes.one of the risk factors for CSVT is hyperhomocysteinemia. In this study we consider clinical profile and surgical outcomes of the patients with Cerebral venous sinus thrombosis (CVST).

\section{Material and Methods}

A retrospective observational study for over a period of 4 years and 3 months from oct' 2013 to feb'2018 in our institute is done. Confirmation of CVST was done by MRI brain venogram of 84 patients. In case of mass effect or herniation decompressive craniectomy was done. All patients were treated according to standard guideline and protocol.

Patients were treated with I. V. fluids to correct lack of hydration, mannitol, anticonvulsant drugs, antibiotics, heparin, conventional, low-molecular weight heparin (LMWH) (if not contraindicated), folic acid and methyl cobalamin supplementations. To avert 
cerebral mass effect and herniation decompressive craniectomy was done. Surgical outcomes of these were analyzed.

Inclusion criteria: All patients determined to have CVST through Magnetic Resonance Imaging of mind venogram/Computer tomography were included in this study

Exclusion criteria: Patient with dural venous sinus thrombosis or acute head injury secondary to primary CNS neoplasia were excluded.

\section{Result}

84 patients diagnosed with CVST were taken as study group in this retrospective observational study, out of 84 patients 51 patients $(60.7 \%)$ were females and 33 patients $(39.2 \%)$ were males with age group of $29 \pm$ 7 years and $39 \pm 10$ years, respectively.

\section{Clinical presentation}

Symptom of headache and vomiting was presented in 79 patients $(94 \%), 13(15 \%)$ had seizures, hemiparesis/hemiplegia in 25 patients $(29.7 \%)$, and fever in $30(35.7 \%)$. Papilledema in $25(29.7 \%)$ on fundoscopy. In CVST most common presenting features are headache and vomiting in patients with $p<$ 0.002 and seizures and hemiplegia follows it.

\section{Contributing factor for CVST}

CVST was most predisposed during peripartum period as $19(32 \%)$ out of 58 female patients were identified with CVST. $35(41.6 \%)$ patients had high serum homocysteine level. Patients with hyperhomocysteinemia of whom 19 were male and 16 were female patients. Confirmation of disease as fever, $22(26.1 \%)$ patients of the total showed symptoms of paranasal sinus (PNS) infections, and/or chronic suppurative otitis media (CSOM). Patients had a history and evidence of lack of hydration in 18 (21.4\%) due to poor intake, fluid loss or because of some other reasons.

\section{Risk factors for the development of CVST}

Table 1: Risk factors for the development of cerebral venous sinus thrombosis

\begin{tabular}{|l|l|}
\hline \multicolumn{1}{|c|}{ Risk factor } & \multicolumn{1}{c|}{ Number of patients n=84 } \\
\hline Oral contraceptive pills & $18(21.4 \%)=18$ female \\
\hline Dehydration & $10(11.9 \%)=7$ male +3 female \\
\hline Infection & $11(13.09 \%)=6$ male +5 female \\
\hline Pregnancy, peripartum & $8(9.5 \%)=8$ female \\
\hline Hyperhomocysteinemia & $23(27.3 \%)=15$ male +8 female \\
\hline Sickle cell disease & $3(3.57 \%)=2$ male $=1$ female \\
\hline Systemic malignancy & $1(1.19 \%)=1$ male \\
\hline Protein c, s deficiency, APLA & $8(9.5 \%)=8$ female \\
\hline Polycythemia vera & $1(1.19 \%)=1$ male \\
\hline Unknown cause & $1(1.19 \%)=1$ male \\
\hline
\end{tabular}

\section{Type of sinus involved in CVST}

$38(45.2 \%)$ out of 84 patients had sagittal sinus thrombosis, $10(11.9 \%)$ out of 84 had multiple sinus thrombosis, and $35(41.6 \%)$ presented with transverse/sigmoid sinus thrombosis. 1 patient who showed up with proptosis involving both the eyes, external ophthalmoplegia and conjunctival congestion with a history of acute or chronic sphenoid and maxillary sinusitis had bilateral cavernous sinus involvement. The mean duration of admission of patients with CVST in our institute was $12 \pm 3$ days.

Table 2: Cerebral venous sinus distribution

\begin{tabular}{|c|c|c|c|c|c|c|}
\hline Cerebral sinus & $\begin{array}{l}\text { Total } \\
\text { patients } \\
\mathrm{N}=84\end{array}$ & $\begin{array}{l}\text { Male } \\
n=33 \\
(39.2 \%)\end{array}$ & $\begin{array}{l}\text { Female } \\
n=51 \\
(60.7 \%)\end{array}$ & $\begin{array}{l}\text { Decompressive } \\
\text { craniectomy } \\
n=25(29.7 \%)\end{array}$ & $\begin{array}{l}\text { Conservative } \\
\mathrm{N}=59 \\
(70.2 \%)\end{array}$ & $\begin{array}{l}\text { Total death }(9.5 \%) \\
\mathrm{N}=8(9.5 \%)\end{array}$ \\
\hline $\begin{array}{l}\text { Superior sagittal } \\
\text { sinus }\end{array}$ & $\begin{array}{c}38 \\
(45.2 \%)\end{array}$ & $\begin{array}{c}11 \\
(33.3 \%)\end{array}$ & $\begin{array}{c}27 \\
(52.9 \%)\end{array}$ & $\begin{array}{c}9 \\
(36 \%)\end{array}$ & $\begin{array}{c}29 \\
(49.1 \%)\end{array}$ & $\begin{array}{c}1 \text { post-op } \\
1 \text { conservative }\end{array}$ \\
\hline $\begin{array}{l}\text { Sigmoid/transverse } \\
\text { sinus }\end{array}$ & $\begin{array}{c}35 \\
(41.6 \%) \\
\end{array}$ & $\begin{array}{c}19 \\
(57.5 \%)\end{array}$ & $\begin{array}{c}16 \\
(31.3 \%)\end{array}$ & $\begin{array}{c}12 \\
(48 \%)\end{array}$ & $\begin{array}{c}23 \\
(38.9 \%)\end{array}$ & 1post-op \\
\hline Cavernous sinus & $\begin{array}{c}1 \\
(1.19 \%)\end{array}$ & $\begin{array}{c}1 \\
(3.03 \%)\end{array}$ & 0 & 0 & $\begin{array}{c}1 \\
(1.9 \%)\end{array}$ & 0 \\
\hline Multiple sinuses & $\begin{array}{c}10 \\
(11.9 \%)\end{array}$ & $\begin{array}{c}2 \\
(6.06 \%)\end{array}$ & $\begin{array}{c}8 \\
(15.6 \%)\end{array}$ & $\begin{array}{c}4 \\
(16 \%)\end{array}$ & $\begin{array}{c}6 \\
(10.1 \%)\end{array}$ & $\begin{array}{c}2 \text { post-op } \\
3 \text { conservative }\end{array}$ \\
\hline
\end{tabular}



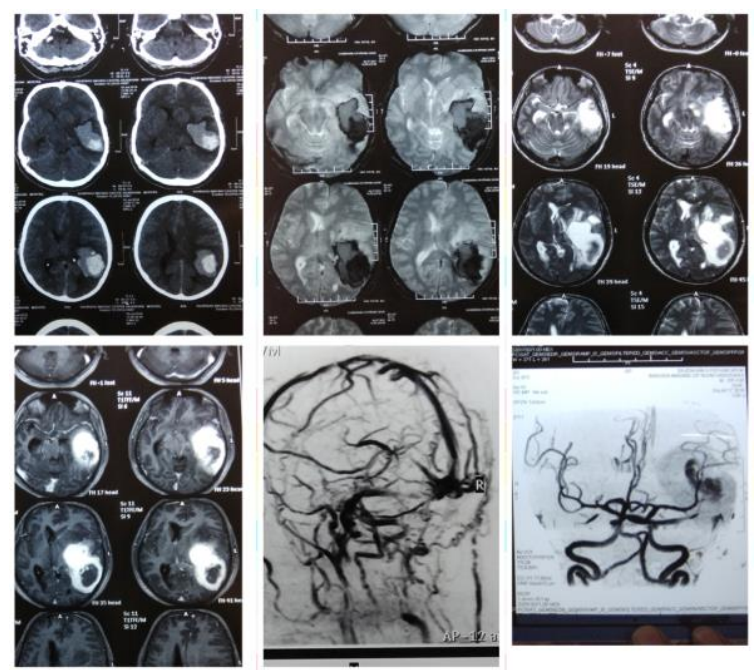

Fig. 1: Images showing left temporal lobe hematoma due to sigmoid sinuses thrombosis and acute left transverse, perilesional edema and midline shift to right side

\section{Outcome of patients with CVST}

Patients who were discharged with partial and/or total recovery from illness were $76(90.4 \%) .59(70.2 \%)$ patients were treated conservatively. 4 patients on conservative treatment died due to various coindisposition factors. Decompressive craniectomy surgery performed in 25 patients $(29.7 \%), 21(84 \%)$ patients showed improvement and 4(16\%) patints succumbed. Total of $8(9.5 \%)$ patients died of which $5(9.8 \%)$ were female with age group of $39.25 \pm$ 11.5years and male patients $3(9.09 \%)$ in number with age group of $58.5 \pm 9$ years. Mortality rate was relatively more in female patients. Majority of patients who died due to the presence of disease had involvement of transverse, sigmoid and/or multiple sinus. Multiple sinus thrombosis had poor outcome and more case fatality rate.

\section{Discussion}

We compared our CVST study experience, inclining elements and featuring its various clinical presentations. $24 \%$ of our population showed Hyperhomocysteinemia, which was more predominant in female population. "According to Lath et al. ${ }^{5}$ in addition to progressive thrombosis, the mortality in CVST, is related to raised intracranial pressure causing transtentorial herniation, a mortality of $27 \%$ was stated by them." In comparision to this our mortality rate was 9.5\% which suggests lower mortality.The decompressive craniectomy with moderate to severe cerebral venous infarcts is an existence sparing technique. In the study, 25 patients (29.7\%) went through decompressive craniectomy out of which 21 (84\%) patients with good outcome and four patients $(16 \%)$ could not survive the disease.

Pfefferkorn et $a l .{ }^{4}$ contemplated that headache being common clinical complaint with CVST in $32(81 \%)$, 9 $(28 \%)$ out of 32 patients, were diagnosed with deep cerebral venous sinus thrombosis (DCVST) and nonisolated DCVST was noted in $23(72 \%)$ patients. In the institutional study done by us, vomiting and headache was noted in 79(94\%) patients. Transverse and/or sigmoid sinus thrombosis was involved in 35 patients $(41.6 \%)$ and superior sagittal sinus thrombosis involved in $38(45.2 \%)$ patients.

Azin et al. ${ }^{6}$ stated that female to male ratio was 3.1:1 in 61 patients with CVST, in which $35.6 \pm 12.1$ years was the average age group, among them $91.8 \%$ of the population showed headache, in which contraceptive consumption $(62.2 \%)$ was the predisposing factor, In our institutional study, $18(21.4 \%)$ female patients with CVST who consumed contraceptive pills, the most common presenting feature was headache noted in 79 patients $(94 \%)$, lack of hydration hyperhomocystemia infection, and peripartum state were the important aggravating risk factors.

Nagaraja et $a l .^{8}$ stated that in India strokes in the youngsters record for almost $30 \%$ of all cases and CVT records for $10 \%-20 \%$ of these cases, in the postpartum period two-thirds of them develop the same, Nagarajan et al. ${ }^{9}$ contemplated total 25 patients with CVST with average age of 35.7 years with a female to male proportion of $3: 1$. Among which, Headache (84\%) and convulsions $(47 \%)$ were the most common clinical signs. In this investigation $51(60.7 \%)$ were females and 33 (39.2\%) were males with $29 \pm 7$ years and $39 \pm 10$ years respectively with CVST in 84 patients, and headache and vomiting being common symptom in $94 \%$ of the patients with hemiplegia in $29.7 \%$. Sigmoid and/or transverse sinus were involved in 35 patients $(41.6 \%)$ and superior sagittal sinus thrombosis in 38 (45.2\%) patients.

Pai et al. ${ }^{11}$ contempleted 612 (39 children, 219 women and 354 men) patients with CVT with headache (62\%), papilledema (62\%), hemiparesis $(48 \%)$, seizures $(31 \%)$, and cranial nerve palsy (7\%). The most common site 
was Superior sagittal sinus thrombosis (74\%) related with lack of hydration, sepsis, pregnancy, and puerperium, in $13 \%$ of population the deaths was because of CVT, with similar result in this study.

Koopman et al. ${ }^{12}$ expressed that oral contraceptive use, pregnancy, or puerperium was causative factor of CVST, more typical with women and hormonal factors may incline to CVT

Rajput et $a l .^{13}$ in their report of CVST noted that acquired hyperhomocysteinemia, apperently because of healthful inadequacies, and was treated with Low Molecular Weight Heparin, followed by warfarin, vit B12/ B6, and folic acid which showed good results. This present study highlighted the causative/risk factors, clinical scenario and possible outcome of patients.

\section{Conclusion}

Early CVST diagnosis is vital and to begin the aggressive treatment of CVST as this can avoid significant morbidity and mortality. 'One third of female population was affected due to oral contraceptive medications, peripartum period has causative factor for CVST'. This study showed noteworthy number of patients affected by CVST in their 20s and 30s. Sigmoid and transverse sinus thrombosis is the most common in males. In female population sagittal sinus is most commonly affected. Decompressive craniectomy is required in patients having sigmoid/transverse sinuses thrombosis.' Headache and vomiting were the most common presenting symptoms followed by seizures and hyperhomocysteinemia was noted in many patients presenting with CVST'. In many patients infection and lack of hydration are the normal predisposing factors for development of CVST. Most susceptibility is seen in peripartum period for the development of CVST in women due to different factors. Decompressive craniectomy is life sparing in patients with mass effect of venous infarction and midline shift, Decompressive craniectomy has great results.

\section{References}

1. Saposnik G, Barinagarrementeria F, Brown RD, Jr, Bushnell CD, Cucchiara B, Cushman M, et al. American Heart Association Stroke Council and the Council on Epidemiology and Prevention. Diagnosis and management of cerebral venous thrombosis: A statement for healthcare professionals from the American Heart Association/American Stroke Association. Stroke 2011;42:1158-92. [PubMed]

2. Prakash C, Bansal BC. Cerebral venous thrombosis. J Ind Acad Clin Med 2000;5:55-61.

3. Nagaraja D, Kruthika-Vinod TP, Christopher R. The prothrombin gene G20210A variant and puerperal cerebral venous and sinus thrombosis in South Indian women. J Clin Neurosci 2007;14:635-8. [PubMed]

4. Pfefferkorn T, Crassard I, Linn J, Dichgans M, Boukobza M, Bousser MG. Clinical features, course and outcome in deep cerebral venous system thrombosis: An analysis of 32 cases. J Neurol 2009;256:1839-45. [PubMed]
5. Lath R, Kumar S, Reddy R, Boola GR, Ray A, Prabhakar $\mathrm{S}$, et al. Decompressive surgery for severe cerebral venous sinus thrombosis. Neurol India 2010;58:3927.[PubMed]

6. Azin $\mathrm{H}$, Ashjazadeh N. Cerebral venous sinus thrombosis - Clinical features, predisposing and prognostic factors. Acta Neurol Taiwan 2008;17:827. [PubMed]

7. Khealani BA, Mapari UU, Sikandar R. Obstetric cerebral venous thrombosis. J Pak Med Assoc 2006;56:4903. [PubMed]

8. Nagaraja D, Sarma GR. Treatment of cerebral sinus/venous thrombosis. Neurol India 2002;50:1146. [PubMed]

9. Nagarajan E, Shankar V. Characteristics of cerebral venous thrombosis in a South Indian Rural Hospital. Int $J$ Med Health Sci 2013;2:298-304.

10. Appenzeller S, Zeller CB, Annichino-Bizzachi JM, Costallat LT, Deus-Silva L, Voetsch B, et al. Cerebral venous thrombosis: Influence of risk factors and imaging findings on prognosis. Clin Neurol Neurosurg 2005;107:371-8. [PubMed]

11. Pai N, Ghosh K, Shetty S. Hereditary thrombophilia in cerebral venous thrombosis: A study from India. Blood Coagul Fibrinolysis 2013;24:540-3.[PubMed]

12. Koopman K, Uyttenboogaart M, Vroomen PC, van der Meer J, De Keyser J, Luijckx GJ. Risk factors for cerebral venous thrombosis and deep venous thrombosis in patients aged between 15 and 50 years. Thromb Haemost 2009;102:620-2. [PubMed]

13. Rajput R, Dhuan J, Agarwal S, Gahlaut PS. Central venous sinus thrombosis in a young woman taking norethindrone acetate for dysfunctional uterine bleeding: Case report and review of literature. J Obstet Gynaecol Can 2008;30:680-3. [PubMed] 